\title{
Emociones asociadas a la primera relación sexual en gays y lesbianas
}

\section{Emotions associated with the first sexual relationship in gays and lesbians}

\author{
Ricardo Sánchez M. ${ }^{1}$, Susana X. Bárcena G. ${ }^{2}$, David, J. Enríquez N. ${ }^{3}$, \\ Sandra I. Muñoz M. ${ }^{4}$ \\ Universidad Nacional Autónoma de México
}

Recibido: $04-05-20$

Aceptado: $12-06-20$

\section{Resumen}

El objetivo del presente trabajo fue evaluar en una muestra de gays y lesbianas, las emociones que experimentaron en su primera relación sexual, así como la relación con el uso de condón, planeación, concenso y edad del debut sexual. Participaron 336 hombres y mujeres, quienes respondieron en línea el cuestionario de Emociones, Estrés y Afrontamiento y algunas preguntas adicionales sobre su primera relación sexual. Se encontraron diferencias significativas en las emociones que experimentan hombres y mujeres, y entre aquellos que usaron condón, planearon y consensuaron su primera relación sexual. Los resultados se discuten en términos de cómo las emociones pueden afectar la vida sexual de gays y lesbianas.

Palabras clave: Debut sexual; gays; lesbianas; condón; emociones.

\begin{abstract}
The objective of the present work was to evaluate in a sample of gays and lesbians, the emotions they experienced in their first sexual relationship, as well as the relationship with condom use, planning, consensus and age of sexual debut. 336 men and women participated, who responded online the Emotions, Stress and Coping questionnaire and some additional questions about their first sexual relationship. Significant differences were found in the emotions experienced by men and women, and among those who used a condom, they planned
\end{abstract}

\footnotetext{
${ }^{1}$ Profesor Titular A de la Universidad Nacional Autónoma de México. Facultad de Estudio Superiores Iztacala. Autor para correspondencia. E-mail: ricardo.sanchez@iztacala.unam.mx

ORCID: https://orcid.org/0000-0003-4268-3025

${ }^{2}$ Profesora Asociada C de la Universidad Nacional Autónoma de México. Facultad de Estudio Superiores Iztacala. E-mail: psicologasbg@gmail.com. ORCID: https://orcid.org/0000-0001-5107-6169

${ }^{3}$ Profesor Titular A de la Universidad Nacional Autónoma de México. Facultad de Estudio Superiores Iztacala. E-mail: david.enriquez@iztacala.unam.mx. ORCID: https://orcid.org/0000-0002-6376-4898

${ }^{4}$ Profesora Asociada C de la Universidad Nacional Autónoma de México. Facultad de Estudio Superiores Iztacala. E-mail: sandra.munoz@iztacala.unam.mx. ORCID: https://orcid.org/0000-0002-9392-5023
}

(C) Los autores. Este artículo es publicado por la Revista de Investigación en Psicología de la Facultad de Psicología, Universidad Nacional Mayor de San Marcos. Este es un artículo de acceso abierto, distribuido bajo los términos de la licencia Creative Commons Atribucion - No Comercia_Compartir Igual 4.0 Internacional. (http://creativecommons.org/licenses/by-nc-sa/4.0/) que permite el uso no comercial, distribución y reproducción en cualquier medio, siempre que la obra original sea debidamente citada. 
and agreed on their first sexual relationship. The results are discussed in terms of how emotions can affect the sex life of gays and lesbians.

Keywords: Sexual debut; gays; lesbians; condom; emotions.

De acuerdo con la Organización Panamericana de la Salud y Organización Mundial de la Salud (OPS/OMS, 2000) la sexualidad se refiere a una dimensión del ser humano en la que están involucrados el sexo, la identidad, el género, la orientación sexual, el erotismo, la vinculación afectiva, el amor y la reproducción. De esta forma cada una de las personas experimentará y expresará diferentes formas de ejercer su sexualidad (Drury y Bukowski, 2013). Según Kar, Choudhury y Singh (2015), el ejercicio de la sexualidad en el adolescente resulta particularmente complicado debido a las tensiones que tiene que experimentar como producto de los cambios biológicos y psicológicos; además, en esta etapa de la vida, la percepción, el pensamiento y la conducta tienden a orientarse hacia el sexo. En este contexto, los jóvenes suelen establecer sus primeras relaciones románticas, las cuales servirán para aprender y experimentar; a través de un avance gradual de intimidad y estimulación, hasta llegar a la penetración (Vargas y Barrera, 2002). En consecuencia, existen muchas probabilidades de consumar la primera relación sexual (Antona, Madrid y Aláez, 2003; Bárcena, Rendón y Robles, 2011); este primer encuentro marcará una transición importante para la mayoría de los adolescentes (Vargas, Henao y González, 2007; Reissing, Andruff y Wentland, 2012) al mismo tiempo que probabiliza situaciones potenciales de riesgo para la salud sexual en el futuro.

La Encuesta Nacional de la Dinámica Demográfica (Instituto Nacional de Estadística y Geografía [INEGI], 2010) señala que en México, el 38\% de los adolescentes entre 12 y 19 años tiene una vida sexual activa, de los cuales en promedio inician a los 15 años y más del $60 \%$ no emplea ningún método para prevenir embarazos o Infecciones de Transmisión Sexual (ITS).

Evitar un embarazo es la principal razón que tienen los jóvenes para usar anticonceptivos (Miño-Worobiej, 2008) y es probable que en la población LGBT, esto no lo conciba como una problemática que pudieran tener; por lo que la principal preocupación sería el contagio de ITS, mismas que generan severas consecuencias para la salud de hombres y mujeres, y debido a que la mayoria son asintomáticas, implican un reto para su detección y tratamiento (Malhotra, 2008). Una ITS que cobra especial relevancia es el VIH dada su prevalencia, consecuencias y debido a que no tiene cura y causa la muerte. De acuerdo con el Programa Conjunto de las Naciones Unidad sobre el VIH/SIDA (ONUSIDA, 2016), desde el comienzo de la pandemia han fallecido más de 35 millones de personas a causa de enfermedades relacionadas con el SIDA. En el caso particular de México (Centro Nacional para la Prevención y Control del VIH y el SIDA [CENSIDA], 2017) tan solo en 2017, se 
han reportado 1,883 nuevos casos de infección, siendo los hombres quienes más casos de VIH y SIDA registran, estableciendo una relación de ocho varones infectados por dos mujeres, representando la principal vía de transmisión, el contacto sexual sin protección.

Además del riesgo potencial de la adquisición de alguna ITS, como el VIH/ SIDA, existen consecuencias a mediano y largo plazo de iniciar la vida sexual a edades tempranas, según lo demuestran algunos estudios, por ejemplo, e ha documentado que el debut sexual temprano se relaciona con mayor número de parejas sexuales a lo largo de la vida (Kastbom, Sydsjö, Bladh, Priebe y Svedin, 2015; Magnusson, Nield y Lapane (2015), con el incremento en la tasa de ITS (Shrestha, Karki y Copenhaver, 2016) y con mayor distrés psicológico (Chiao y Ksobiech, 2015). Debido a a todos los riesgos potenciales y a sus consecuencias, así como a la baja frecuencia del uso de métodos para prevenir un embarazo o ITS en la primera relación sexual (INEGI, 2010) se han desarrollado diversas investigaciones para identificar aquellas condiciones que precipitan a los jóvenes a tener su primer encuentro sexual.

Variables psicosociales como una percepción de poco control personal (Gi1liam, Berlin, Kozloski, Hernández y Grundy, 2007), baja autoestima (Day, 1992), valores tradicionales - por ejemplo machismo- y desligados de la religión y la moral (Cummings, Auerswald y Ott, 2014) están asociadas con el inicio precoz de la vida sexual. La familia juega un papel activo en la socialización sexual de los hijos (Fontenberry, 2013) y en consecuencia en la promoción de conductas sexuales, por ejemplo, la falta de expectativas parentales sobre el logro educativo y una baja frecuencia de mensajes alusivos sobre el sexo y el embarazo pre matrimonial (Gilliam et al., 2007), prácticas parentales y estilos de crianza permisivos (Amoateng, 2017; Climent, 2009; Gravel, Young, Darzi, Olavarria-Turner y Ming-Lee, 2016) y la baja frecuencia de monitoreo parental (Longmore, Manning y Giordano, 2001) predisponen al contacto sexual temprano.

También, existen condiciones contextuales que tienen influencia, por ejemplo, Upchurch, Mason, Kusunoki y Kriechbaum (2004) y Longmore, Manning y Giordano (2001) señalan que ambientes con altos índices de desempleo y pobreza predisponen situaciones para que los adolescentes consumen su primer encuentro sexual. Respecto a los factores sociodemográficos, se ha identificado que el nivel educativo bajo, el abandono escolar y una condición laboral activa son un perfil asociado con un debut sexual temprano (Nigatu, Seme, Fituma y Segni, 2018). También se han reportado resultados diferenciales por sexo, por ejemplo, los hombres, en comparación con las mujeres, inician su vida sexual a edades más tempranas; siendo los que tienen sexo con personas de su mismo sexo, quienes más temprano inician su vida sexual (Brown, Masho, Perera, Mezuk y Cohen, 2015). 
La experiencia de la primera relación sexual se complejiza cuando se consideran las variables situacionales bajo las cuales ocurre el encuentro; por ejemplo, estar excitado, en estado etílico o bajo el efecto de drogas disminuye las posibilidades de usar preservativo (Carpenter, Janssen, Graham, Vorst y Wicherts, 2008; Nigatu, Seme, Fituma y Segni, 2018; Piña, González, Molina y Cota, 2003), lo mismo sucede cuando se experimenta amor romántico por la pareja (Goldmeir y Richardson, 2005); además como señalan Lieberman, Goldfarb, Kwiatkowski y Santos (2017), en su mayoría los jóvenes que no planean su primera relación sexual, tienden a no usar el condón, por lo que pareciera que existen diversos procesos fisiológicos y psicológicos que tiene un papel importante para probabilizar conductas protegidas; esa convergencia puede encontrarse en las emociones que experimentan; entendiendo a la emoción como una reacción psicofisiológica que representa modos de adaptación a ciertos estímulos del individuo cuando percibe un objeto, persona, lugar, suceso o recuerdo importante (Levenson, 1994).

Se ha documentado que cuando los jóvenes usan condón en su primera relación sexual es más probable que lo hagan en sus encuentros sexuales subsecuentes (Juárez y Gayet, 2005). Describir las condiciones de este evento resulta fundamental, ya que la forma en que la persona actúa en las siguientes situaciones, se va configurando desde la primera relación sexual. En este sentido, identificar las emociones que experimentan durante la primera relación sexual podría contribuir en la compresión de aquellos factores asociados con la conducta sexual temprana y el uso de métodos de protección, como el condón.

Con base en lo anterior, las emociones se definen como una perturbación compleja que incluye tres componentes principales: el afecto subjetivo (que incluye la evaluación cognitiva), los cambios fisiológicos y las acciones; de tal forma que la calidad o intensidad de la emoción y su impulso de acción dependen de una evaluación cognitiva del significado (Lazarus, 1998); de tal forma que las emociones pueden precipitar comportamientos particulares, mismos que puede estar orientados hacia la prevención o el riesgo; por ejemplo, Miner, Dickenson y Coleman (2019) demostraron que existe una relación causal entre el afecto y la conducta sexual; los resultados muestran que la intensidad de las emociones positivas y negativas precipitan, de manera diferencial, al ejercicio de diferentes comportamientos sexuales. Así, las emociones podrían contribuir, junto con otras variables, de naturaleza conductual y psicosocial a explicar cómo y en qué situaciones particulares, las emociones predisponen a conductas que incrementan el riesgo de contagio por VIH o ITS, o por el contrario, permiten adoptar comportamiento sexualmente preventivos.

En este sentido identificar las emociones en está situación particular podría brindar información útil sobre como las reacciones emocionales, en esta situación particular, pueden influir sobre la conducta preventiva; lo anterior implica ir más 
allá de describir situaciones, características sociodemográficas o variables cognitivas -como percepción, valores, expectativas, creencias- para complementar el análisis con el papel que juegan los procesos psicofisiológicos en la regulación de la conducta preventiva en el primer encuentro sexual, si bien, se esperaría que dicha evaluación se realizará en un momento específico y lo más pronto posible para evitar problemas de memoria por parte del respondiente, en psicología no siempre es posible hacerlo (Dang, King y Inzlicht, 2020; Lagunes, 2017); de tal forma que como mencionan Sánchez, Enríquez y Rosales (2019), la experiencia que se adiquiere a lo largo de la vida puede influir en la presencia o ausencia de conductas sexuales de riesgo, y el momento en particular en el que se evalua es determinante para saber como intervenir en ese momento; por ejemplo, existe evidencia de cómo influye la primera relación sexual en los encuentros sexuales posteriores, y cómo a partir de la evaluación de ese primer encuentro se pueden tomar decisiones para promover la salud sexual, tanto en personas que no han iniciado ese primer encuentro, como en personas que ya iniciaron (Brown et al., 2015, Lai et al., 2018; Lowry, Dunville, Robin y Kann, 2017); asímismo se ha demostrado el efecto que tienen las emociones en conductas de riesgo, y de cómo las emociones varían con el paso del tiempo y cómó influyen en la conducta actual (Caballero, Carrera, Sánchez, Muñoz y Blanco; 2003).

También es necesario poner atención a la características de la población, principalmente en contextos de riesgo, tal es el caso de la comunidad de lesbianas, gays, bisexuales y transgénero (LGBT), dado que se deben considerar los efectos de la cultura, ideología y normas sociales que permean el comportamiento de una persona con una orientación sexual diferente a la heterosexual (Delgado, Gordon, y Schnarrs, 2016; Lowry, Johns, Robin y Kann, 2017; Riggs y Treharne, 2017). En un contexto social de hegemonía heterosexual las personas homosexuales se ubican en una posición social desventajosa y estigmatizada (Herek, 2007), lo cual deriva en un alto nivel de estrés debido a aspectos relacionados con la homofobia, el rechazo, discriminación y violencia (Iniewicz, Sałapa, Wrona y Marek, 2017; Pollard, Nadarzynski y Llewellyn, 2018), alta prevalencia de problemas de desajuste emocional (Sattler, Franke y Christiansen, 2017; Vicuña, 2017) y mayor frecuencia de conductas sexuales de riesgo (Chenchang et al., 2017; Lowry, Dunville, Robin y Kann, 2017).

Ahora bien, la literatura señala que los hombres homosexuales en comparación con las mujeres homosexuales tienen más riesgo de adquirir VIH; esto debido a que al practicar sexo anal hay mayor concentración viral y mayor capacidad de transmisión del semen del varón con lesiones debidas a la penetración anal (VallMayans, 2006). De igual forma reportan una peor evaluación de los lazos que mantienen con la sociedad y mayores problemas en su salud mental (Gómez y Barrientos, 2012). Autores como Herek (2007) y Whitley (2001) explican que esto puede deberse a que existe mayor rechazo y hostilidad hacia la homosexualidad 
masculina, al tiempo que existe una mayor trangresión de los estereotipos de género en ellos, ya que los hombres gays son pensados como afeminados, mientras que las mujeres lesbinas son vistas de forma masculina (Carrier, 1976).

Otro problema que suele presentarse entre los gays y lesbianas es el tema del abuso sexual, entendido en este estudio, como el hecho de no consentir tener relaciones sexuales. Trenchad y Warren (1984) identificaron que uno de cada tres de 416 jóvenes gays y lesbianas fueron víctimas de abuso sexual por motivo de su orientación sexual. Una de las mayores dificultades que tiene esta población es pedir ayuda ya que a menudo también experimentan aislamiento social, esta variable cobra relevancia debido a que en hombres gay los encuentros sexuales no concensuados incrementan el riesgo para la adquisición del VIH (Arreola, Neilands, Pollack, Paul y Catania, 2008).

Con base en lo anterior, el objetivo del presente trabajo fue evaluar en una muestra de gays y lesbianas, las emociones experimentadas en la primera relación sexual, así como su relación con el uso de condón, planeación, consenso y edad de inicio de la vida sexual.

\section{MÉTODO}

\section{Muestra}

336 hombres y mujeres participaron en la presente investigación, a través de un muestreo por conveniencia (Kerlinger y Lee, 2002), se invitó a responder un cuestionario en línea a los usuarios de un portal web dirigido la comunidad LGBT. El $62.5 \%$ de los participantes fueron gays y el $37.5 \%$ lesbianas. Se tomaron como criterios de inclusión ser mayores de edad, haber tenido relaciones sexuales alguna vez en su vida y se excluyeron los casos que no respondieron en su totalidad la evaluación. La edad promedio de los hombres fue de 25.20 años $(\mathrm{DE}=7.82)$, mientras que de las mujeres fue de 21.90 años $(\mathrm{DE}=6.42)$. El $87.8 \%$ reportaron ser solteros y el resto se asumieron como casados o en unión libre; el 59.2\% informó tener estudios de universidad, $15.2 \%$ carrera técnica, $10.1 \%$, secundaria, $9.5 \%$ primaria y $6 \%$ posgrado.

\section{Instrumentos}

Se aplicó el cuestionario de Emociones, Estrés y Afrontamiento (CEEA) de Vega, Muñoz, Berra, Nava y Gómez (2012), el cual evalúa 15 emociones en situaciones estresantes y placenteras que son descritas por el participante en una escala de cero (nada) a cuatro (mucho). El alfa de Cronbach reportado para este instrumento es de 0.84 , sin embargo, debido al objetivo de la investigación, se realizó una adaptación al cuestionario y se solicitó a los participantes que informaran su experiencia en términos de la primera relación sexual. Adicionalmente se evaluó el patrón 
de comportamiento sexual, que incluyó uso de condón, planeación y concenso en la primera relación sexual, así como la edad del debut sexual.

\section{Procedimiento}

Se realizó un formulario electrónico a través del cual se cargaron las preguntas del CEEA y del patrón de comportamiento sexual, se estableció un convenio con el editor del portal \#Soy Homosensual para que a través de su página web se difundiera; el cual permaneció disponible en un periodo de dos meses. Aquellas personas que deseaban participar, se les indicó que la información proporcionada sería anónima y confidencial; el cuestionario estuvo configurado para agradecer a los participantes al momento de enviar sus respuestas; en promedio tardaron 30 minutos en responder las preguntas.

\section{Analisis de resultados}

Se utilizó el paquete estadístico SPSS (Statistical Package for the Social Sciences) para el tratamiento estadístico de los datos. Se realizó una comparación entre grupos utilizando la prueba $t$ de student para muestras no relacionadas en aquellas variables numéricas y la ji cuadrada para las variables categóricas; por último se realizó un análisis de asociación estimado el coeficiente de correlacón de Pearson (r), se calculó la $d$ de Cohen y su interpretación se basó en Cárdenas y Arancibia (2014), que señalan que el tamaño del efecto se considera .20 pequeño, .50 mediano y .80 grande.

\section{RESULTADOS}

Se realizó un análisis de diferencias con la ji cuadrada para identificar, si había diferencias en gays y lesbianas en cuanto al uso del condón, planeación, concenso en la primera relación sexual y edad del debut sexual, y no se encontraron diferencias estadísticamente significativas. En términos generales se encontró que el 48.1\% uso condón en su primera relación sexual, el 35.9\% la planeó, el 9\% reportó que su primer encuentro sexual no fue consensuado y la edad de inicio de relaciones sexuales fue a los 17 años $(\mathrm{SD}=4.03)$.

La Tabla 1 muestra los resultados obtenidos de las emociones experimentadas en la primera relación sexual, haciendo la comparación por hombres y mujeres en términos de su orientación sexual, se encontraron diferencias estadísticamente significativas en cuatro emociones negativas y en una positiva; los hombres reportaron haber sentido mayor ansiedad, temor, culpa y vergüenza; mientras que las mujeres más amor. Grosso modo se observa que el tamaño del efecto es bajo, pero en aquellas emociones en las que hubo diferencias significativas el tamaño del efecto es mediano. 
Tabla 1

Emociones experimentadas por gays y lesbianas en primera relación sexual

\begin{tabular}{|c|c|c|c|c|c|c|c|c|}
\hline \multirow{2}{*}{$\begin{array}{l}\text { Emoción } \\
\text { Ira }\end{array}$} & \multicolumn{2}{|c|}{$\begin{array}{c}\text { Gays } \\
(n=210)\end{array}$} & \multicolumn{2}{|c|}{$\begin{array}{c}\text { Lesbianas } \\
(n=126)\end{array}$} & \multirow{2}{*}{$\begin{array}{c}\mathbf{t} \\
.972\end{array}$} & \multirow{2}{*}{$\begin{array}{c}\text { gl } \\
289\end{array}$} & \multirow{2}{*}{$\begin{array}{l}\text { Sig. } \\
.332\end{array}$} & \multirow{2}{*}{$\begin{array}{c}\begin{array}{c}\text { d de } \\
\text { cohen }\end{array} \\
.09\end{array}$} \\
\hline & .41 & .865 & .31 & .76 & & & & \\
\hline Ansiedad & 1.75 & 1.120 & 1.38 & 1.117 & 2.716 & 293 & .007 & .36 \\
\hline Temor & 1.85 & 1.046 & 1.44 & 1.113 & 3.162 & 295 & .002 & .41 \\
\hline Culpa & 1.24 & 1.223 & .90 & 1.154 & 2.337 & 295 & .020 & .33 \\
\hline Vergüenza & 1.63 & 1.148 & 1.25 & 1.111 & 2.785 & 295 & .006 & .38 \\
\hline Tristeza & .62 & 1.046 & .55 & .980 & .509 & 293 & .611 & .06 \\
\hline Envidia & .11 & .445 & .05 & .254 & 1.396 & 290 & .164 & .06 \\
\hline Celos & .14 & .522 & .10 & .456 & .593 & 290 & .554 & .03 \\
\hline Felicidad & 1.66 & 1.093 & 1.70 & 1.164 & -.296 & 297 & .767 & .04 \\
\hline Orgullo & .93 & 1.071 & .84 & 1.105 & .686 & 290 & .493 & .09 \\
\hline Alivio & .97 & 1.073 & .85 & 1.058 & .955 & 290 & .340 & .12 \\
\hline Esperanza & .75 & 1.039 & .87 & 1.113 & -.897 & 289 & .370 & .11 \\
\hline Amor & 1.28 & 1.223 & 1.64 & 1.316 & -2.315 & 293 & .021 & .35 \\
\hline Gratitud & .92 & 1.110 & 1.02 & 1.246 & -.703 & 288 & .483 & .10 \\
\hline Compasión & .49 & .910 & .43 & .851 & .535 & 289 & .593 & .05 \\
\hline
\end{tabular}

Para identificar si había diferencia entre las emociones de quienes utilizaron, y no, el condón en el primer encuentro sexual, se llevó a cabo un contraste, utilizando la t de student para muestras no relacionadas. En la Tabla 2 se observa que únicamente se encontraron diferencias estadísticamente significativas en dos emociones positivas; es decir, aquellos que no usaron condón experimentaron más amor y mayor felicidad; sin embargo hacer un análisis respecto al tamaño del efecto se observa que es bajo.

En la Tabla 3 se muestran los resultados del análisis comparativo entre quienes planearon, y no, su primera relación sexual y las emociones que experimentaron. Los participantes que no planearon su primer encuentro sexual experimentaron ira, culpa, vergüenza y tristeza en contraste con quienes si la planificaron; además, éstos últimos informaron sentir felicidad, alivio, amor y gratitud, siendo significativas estas diferencias. El tamaño del efecto en aquellas emociones que no fueron significativas es bajo, así como en las emociones de ira, vergüenza, tristeza y alivio; se encontró un tamaño del efecto mediano en culpa, felicidad y gratitud, y un efecto grande en amor. 
Tabla 2

Emociones experimentadas por gays y lesbianas sobre el uso de condón en primera relación sexual

\begin{tabular}{lcccccccc}
\hline Emoción & \multicolumn{2}{c}{$\begin{array}{c}\text { Sí uso } \\
(\mathbf{n = 1 2 5})\end{array}$} & \multicolumn{2}{c}{$\begin{array}{c}\text { No uso } \\
(\mathbf{n = 1 3 5})\end{array}$} & $\mathbf{t}$ & gl & Sig. & $\begin{array}{c}\text { d de } \\
\text { cohen }\end{array}$ \\
\hline Ira & .41 & .858 & .37 & .811 & .405 & 250 & .686 & 0.04 \\
Ansiedad & 1.66 & 1.115 & 1.68 & 1.114 & .186 & 251 & .853 & 0.02 \\
Temor & 1.77 & 1.021 & 1.76 & 1.080 & .021 & 253 & .983 & 0.03 \\
Culpa & 1.12 & 1.234 & 1.25 & 1.198 & .860 & 253 & .391 & 0.13 \\
Vergüenza & 1.52 & 1.130 & 1.65 & 1.109 & .889 & 253 & .375 & 0.12 \\
Tristeza & .67 & 1.087 & .50 & .917 & .344 & 252 & .180 & 0.16 \\
Envidia & .11 & .409 & .09 & .423 & .397 & 250 & .692 & 0.02 \\
Celos & .14 & .501 & .12 & .498 & .318 & 248 & .751 & 0.02 \\
Felicidad & 1.58 & 1.075 & 1.84 & 1.109 & 1.928 & 256 & .05 & 0.26 \\
Orgullo & .89 & 1.107 & 1.02 & 1.104 & .925 & 249 & .356 & 0.12 \\
Alivio & .91 & 1.037 & .97 & 1.082 & .442 & 249 & .659 & 0.05 \\
Esperanza & .77 & 1.062 & .81 & 1.060 & .288 & 248 & .773 & 0.03 \\
Amor & 1.28 & 1.253 & 1.56 & 1.256 & 1.749 & 251 & .05 & 0.27 \\
Gratitud & .90 & 1.146 & 1.01 & 1.158 & .727 & 247 & .468 & 0.10 \\
Compasión & .43 & .898 & .53 & .898 & .843 & 248 & .400 & 0.09 \\
\hline
\end{tabular}

Tabla 3

Emociones experimentadas por gays y lesbianas sobre la planeación en primera relación sexual

\begin{tabular}{|c|c|c|c|c|c|c|c|c|}
\hline \multirow{2}{*}{$\begin{array}{l}\text { Emoción } \\
\text { Ira }\end{array}$} & \multicolumn{2}{|c|}{$\begin{array}{c}\text { Si la planeó } \\
(n=97)\end{array}$} & \multicolumn{2}{|c|}{$\begin{array}{l}\text { No la planeó } \\
(n=173)\end{array}$} & \multirow{2}{*}{$\begin{array}{c}\mathbf{t} \\
2.504\end{array}$} & \multirow{2}{*}{$\begin{array}{c}\mathbf{g l} \\
259\end{array}$} & \multirow{2}{*}{$\begin{array}{l}\text { Sig. } \\
.013\end{array}$} & \multirow{2}{*}{$\begin{array}{c}\begin{array}{c}\text { d de } \\
\text { cohen }\end{array} \\
0.26\end{array}$} \\
\hline & .22 & .605 & .49 & .932 & & & & \\
\hline Ansiedad & 1.56 & 1.054 & 1.76 & 1.150 & 1.373 & 260 & .171 & 0.19 \\
\hline Temor & 1.70 & 1.027 & 1.85 & 1.072 & 1.090 & 262 & .277 & 0.14 \\
\hline Culpa & .88 & 1.050 & 1.39 & 1.278 & 3.287 & 262 & .001 & 0.50 \\
\hline Vergüenza & 1.42 & 1.078 & 1.70 & 1.142 & 1.972 & 262 & .050 & 0.28 \\
\hline Tristeza & .37 & .839 & .74 & 1.100 & 2.887 & 261 & .004 & 0.37 \\
\hline Envidia & .06 & .320 & .12 & .451 & 1.091 & 259 & .276 & 0.05 \\
\hline Celos & .09 & .463 & .15 & .511 & .811 & 257 & .418 & 0.05 \\
\hline Felicidad & 2.00 & .984 & 1.53 & 1.123 & 3.411 & 265 & .001 & 0.46 \\
\hline Orgullo & 1.05 & 1.124 & .87 & 1.083 & 1.272 & 258 & .204 & 0.18 \\
\hline Alivio & 1.16 & 1.085 & .81 & 1.023 & 2.611 & 258 & .010 & 0.35 \\
\hline Esperanza & .86 & 1.038 & .74 & 1.072 & .873 & 257 & .383 & 0.11 \\
\hline Amor & 1.96 & 1.175 & 1.11 & 1.208 & 5.531 & 260 & .000 & 0.85 \\
\hline Gratitud & 1.26 & 1.242 & .77 & 1.057 & 3.342 & 256 & .001 & 0.48 \\
\hline Compasión & .60 & .961 & .40 & .856 & 1.710 & 257 & .088 & 0.19 \\
\hline
\end{tabular}


En la Tabla 4 se observa la comparación entre quienes tuvieron su primera relación consensuada, y quienes no. Se observa que cuando la primera relación sexual es no concensuada se experimentó ira, temor, culpa, vergüenza y tristeza; sin embargo, cuando la decisión fue por acuerdo común los participantes manifestaron presentar felicidad, amor y gratitud. Las diferencias entre los puntajes por grupo para cada una de estas emociones fueron estadísticamente significativas. En las emociones en las que no se encontró diferencias significativas el tamaño del efecto es pequeño; en las emociones ira, temor, culpa, vergüenza, tristeza y gratitud, el efecto es mediano; y en las emociones felicidad y amor, el efecto es grande.

\section{Tabla 4}

Emociones experimentadas por gays y lesbianas sobre el concenso en la primera relación sexual

\begin{tabular}{lcccccccc}
\hline Emoción & \multicolumn{2}{c}{$\begin{array}{c}\text { Si la consensuó } \\
(\mathrm{n}=34)\end{array}$} & \multicolumn{2}{c}{$\begin{array}{c}\text { No la consensuó } \\
(\mathrm{n}=34)\end{array}$} & $\mathbf{t}$ & gl & Sig. & $\begin{array}{c}\text { d de } \\
\text { cohen }\end{array}$ \\
\hline Ira & .34 & .774 & .96 & 1.224 & -3.437 & 54 & .001 & 0.61 \\
Ansiedad & 1.68 & 1.092 & 1.83 & 1.370 & -.601 & 55 & .549 & 0.14 \\
Temor & 1.77 & 1.028 & 2.17 & 1.204 & -1.778 & 55 & .050 & 0.39 \\
Culpa & 1.16 & 1.203 & 1.83 & 1.274 & -2.616 & 54 & .009 & 0.67 \\
Vergüenza & 1.55 & 1.116 & 2.25 & 1.032 & -2.964 & 54 & .003 & 0.70 \\
Tristeza & .55 & .983 & 1.22 & 1.313 & -2.991 & 54 & .003 & 0.66 \\
Envidia & .10 & .416 & .13 & .344 & -.368 & 54 & .714 & 0.03 \\
Celos & .14 & .514 & .04 & .209 & .862 & 54 & .390 & 0.09 \\
Felicidad & 1.77 & 1.062 & .96 & 1.197 & 3.538 & 55 & .001 & 0.81 \\
Orgullo & .97 & 1.105 & .70 & 1.063 & 1.123 & 54 & .262 & 0.27 \\
Alivio & .95 & 1.055 & .75 & 1.032 & .880 & 54 & .379 & 0.19 \\
Esperanza & .79 & 1.043 & .74 & 1.176 & .205 & 53 & .838 & 0.04 \\
Amor & 1.49 & 1.253 & .58 & 1.018 & 3.419 & 55 & .001 & 0.90 \\
Gratitud & 1.00 & 1.157 & .39 & .941 & 2.443 & 53 & .015 & 0.60 \\
Compasión & .50 & .917 & .17 & .650 & 1.674 & 53 & .095 & 0.32 \\
\hline
\end{tabular}

Por último, sobre la edad del debut sexual se realizó un análisis de correlación para determinar si había asociación entre las emociones y la edad de inicio de la vida sexual. Considerando toda la muestra, se encontraron dos correlaciones negativas y una positiva; es decir, entre más tempranamente inicien su vida sexual, experimentan mayor culpa y vergüenza; mientras que quienes inician debutan sexualmente a edades más tardias, sienten mayor sensación de alivio. No hubo correlaciones para el grupo de lesbianas y solamente en gays para culpa y alivio (ver Tabla 5). Respecto al tamaño del efecto el valor de $r$ de Pearson se inteprerpreta considerando los mismo puntajes de $d$ de Cohen; en aquellas variables en las que se encontrón una relación signiticativa el tamaño del efecto es 
pequeño, adicionalmente se obtuvo el porcentaje de la varianza compartida y en términos generales se explica alrededor del $30 \%$.

\section{DISCUSIÓN}

El objetivo de la presente investigación fue evaluar en una muestra de gays y lesbianas las emociones que experimentaron sobre su primera relación sexual, al hacer un análisis de diferencias por sexo se encontró que los hombres experimentan más emociones negativas, como ansiedad, temor, culpa y vergüenza, a diferencia de las mujeres; esto puede relacionarse con lo que menciona Iniewicz, Sałapa, Wrona y Marek (2017) sobre los efectos de la cultura, en donde debido a una predominancia de población heterosexual, se hace más dificil el proceso de adaptación para los hombres gays al no cumplir con los estereotipos sociales (Riggs y Treharne, 2017). En el caso particular de las mujeres se encontró que ellas experimentaron más amor, dada la valencia afectiva de la emoción, pudiera parecer que es sencillo para ellas iniciarse en sus relaciones sexuales con alguien de su mismo sexo. Autores como Herek (2007) y Whitley (2001) explican que menor rechazo y hostilidad hacia la homosexualidad femenina, debido quizás a que los hombres gays son vistos como afeminados, mientras que las mujeres lesbianas son vistas de forma masculina (Carrier, 1976).

Tabla 5

Relación entre emociones y edad del inicio de relaciones sexuales.

\begin{tabular}{|c|c|c|c|c|c|c|}
\hline Emoción & $\begin{array}{l}\text { Toda muestra } \\
\quad(n=336)\end{array}$ & $\begin{array}{c}\text { Tamaño del } \\
\text { efecto }\left(r^{2}\right)\end{array}$ & $\begin{array}{c}\text { Gays } \\
(n=210)\end{array}$ & $\begin{array}{c}\text { Tamaño del } \\
\text { efecto }\left(\mathbf{r}^{2}\right)\end{array}$ & $\begin{array}{c}\text { Lesbianas } \\
(n=126)\end{array}$ & $\begin{array}{c}\text { Tamaño del } \\
\text { efecto }\left(r^{2}\right)\end{array}$ \\
\hline Ira & -.035 & 7 & -.027 & 5.4 & -.057 & 11.4 \\
\hline Ansiedad & -.044 & 8.8 & -.033 & 6.6 & -.062 & 12.4 \\
\hline Temor & -.060 & 12 & -.112 & 22.4 & .094 & 18.8 \\
\hline Culpa & $-.166^{*}$ & $33.2 * * *$ & $-.187 * *$ & $37.4 * * *$ & -.103 & 20.6 \\
\hline Vergüenza & $-.149 * *$ & $29.8^{* * *}$ & -.135 & -27 & -.186 & 37.2 \\
\hline Tristeza & .000 & 0 & .013 & 2.6 & -.037 & 7.4 \\
\hline Envidia & .059 & 11.8 & .043 & 8.6 & .148 & 29.6 \\
\hline Celos & .038 & 7.6 & .050 & 10 & .011 & 2.2 \\
\hline Felicidad & .028 & 5.6 & .010 & 2 & .076 & 15.2 \\
\hline Orgullo & .117 & 23.4 & .104 & 20.8 & .160 & 32 \\
\hline Alivio & $.168^{*}$ & $33.6^{* * *}$ & $.180 * *$ & $36^{* * *}$ & .144 & 28.8 \\
\hline Esperanza & .090 & 18 & .122 & 24.4 & .009 & 1.8 \\
\hline Amor & .088 & 17.6 & .074 & 14.8 & .124 & 24.8 \\
\hline Gratitud & .117 & 23.4 & .090 & 18 & .194 & 38.8 \\
\hline Compasión & .051 & 10.2 & .055 & 11 & .040 & 8 \\
\hline
\end{tabular}

$* .001$

$* * 05$

*** Porcentaje de la varianza compartida entre las variables. 
Cerca de la mitad de hombres y mujeres reportaron que no usaron condón en la primera relación sexual, más del $60 \%$ no lo planeó, lo cual se torna relevante porque estuvieron expuestos, desde este primer encuentro, a infectarse por VIH, poniendose en una situación de riesgo. Se debe destacar que el 9\% reportó que su primera relación sexual no fue consensuada, si bien no se preguntó directamente por un acto de abuso sexual, es un indicador que debe considerarse, debido a que el porcentaje es importante y como consecuencia se pueden generar problemas emocionales (Sattler, Franke y Christiansen, 2017) y favorecer las condiciones psicológicas para el desarrollo de conductas de riesgo (Chenchang et al. 2017).

Las emociones asociadas al uso del condón en la primera relación sexual fueron amor y felicidad, esto significa que para quienes lo usaron sintieron emociones de valencia positiva, valdría la pena evaluar si el sentir estas emociones puede asociarse con el uso del preservativo en posteriores relaciones sexuales, con el propósito de identificar cómo pueden ayudar en el proceso de toma de decisiones (Lai Tong Lee, Loke, Tsz Man Hung y Sobel, 2018).

Sobre las emociones experimentadas en la planeación de la primera relación sexual, se encontró que quienes no la planearon sintieron más ira, culpa, vergüenza y tristeza; mientras que quienes lo hicieron experimentaron más felicidad, alivio, amor y gratitud; esto es relevante, primero, debido a la carga emocional que tiene iniciar la vida sexual activa, y segundo, por los diversos factores que propiciaron el inicio de la vida sexual sin haberlo planeado (Tenkorang y MatickaTyndale, 2014).

Aquellos que tuvieron su primera relación sexual no consensuada, se encontró que la ira, temor, culpa, vergüenza y tristeza, fueron emociones que estuvieron asociadas con ellos; mientras que, quienes si lo tuvieron, experimentaron emociones positivas como felicidad, amor y gratitud. La literatura señala que esta población es más vulnerable a ser víctima de abuso sexual por motivo de su orientación sexual (Trenchad y Warren, 1984). Igualmente, esta variable cobra relevancia particularmente en los hombres gays, debido a que en ellos este tipo de encuentros incrementan el riesgo para la adquisición del VIH (Arreola, Neilands, Pollack, Paul y Catania, 2008). Se debe poner atención en aquellas personas que no tuvieron consenso en su primera relación sexual para ayudar y evitar problemas de desajuste emocional, reducir el estrés que les generó y que puedan generar estrategias de afrontamiento más adecuadas sobre su conducta sexual posterior (Chiao y Ksobiech, 2015; Shrestha, Karki y Copenhaver, 2016).

Por último, se realizó un análisis de relación entre la edad del debut sexual y las emociones experimentadas, al hacer el análisis en conjunto de hombres y mujeres se encontró que a menor edad del debut sexual, experimentan más culpa y vergüenza; mientras que quienes inician su vida sexual a edades más tardias, sienten alivio; sin embargo, al hacer la comparación dividiendo a hombres y mujeres, 
estos resultados se presentan sólo en hombres; parece ser que la edad en la que inician las mujeres no tuvo una relación con las emociones que experimentaron.

La edad de inicio de las relaciones sexuales, es relevante dado que quienes inician a edades más tempranas tienden a desarrollar conductas de más riesgo que ponen en riesgo su salud sexual (Kastbom, Sydsjö, Bladh, Priebe y Svedin, 2015; Shrestha, Karki y Copenhaver, 2016). Algo relevante a mencionar es que en el caso de los hombres, las emociones tienen una carga más negativa, si es que ellos inician su vida sexual a edades más tempranas, si se relaciona, con otros factores, como la falta de planeación y no usar condón; aunado al cuestionamiento de las normas sociales, será díficil que hombres gays puedan generar estrategias que les pemita afrontar dicha situación, así como encuentros sexuales posteriores.

De igual forma en cada uno de los análisis presentados, se realizó un análisis del tamaño del efecto, esto con la intención de no sólo conocer si existen diferencias entre los puntajes obtenidos de gays y lesbianas, sino de conocer la magnitud de esas diferencias, además de que permite en futuras investigaciones comparar estos resultados con otros, de tal forma que se puede tener evidencias de validez adicionales (Cárdenas y Arancibia, 2014). Con base en lo anterior, se evalúo el tamaño del efecto, independientemente de si el resultado presentó diferencias estadísticamente significativas, generalmente en aquellas variables en las que se presentaron estas diferencias el tamaño del efecto fue bajo. En cambio en aquellas en las si las hubo, se encontraron diferentes interpretaciones.

Por ejemplo, en le caso de la primera relación sexual y del uso del condón, la diferencia en la percepción de las emociones tuvo un efecto pequeño, pareciera ser que para ellos las emociones que experimentaron son similares, por lo que es necesario identificar si esto se debe la misma condición de orientación sexual, por lo que se requiere en futuras investigaciones, comparar grupos en términos de su orientación sexual para identificar si existen estas diferencias.

En cambio si se encontró un tamaño del efecto mediano y grande respecto a las emociones experimentadadas respecto a la planeación de su primer encuentro sexual, esto es relevante si se considera que las emociones negativas predominan en quienes no la planearon y las emociones positivas en quienes si la planearon, de tal forma que como se muestra en diversas investigaciones, la importancia que tiene que las personas planeen su primer encuentro sexual para reducir conductas de riesgo (Chenchang et al., 2017), y probablemente las emociones negativas.

Un punto importante dentro de los resultados del tamaño del efecto, tiene que ver con las emociones experimentadas respecto al consenso sexual, se encontraron tamaños del efecto medianos y grandes, de tal forma que en aquellos que si fue 
consensuada hay emociones más positivas, esto es relevante dado que es probable que haya un contexto de violación, de tal forma que como mencionan Brown et al (2015) y Chiao y Ksobiech (2015), será más probable que en sus próximas relaciones sexuales haya conductas de riesgo o problemas de salud mental, de tal forma que futuras investigaciones se requiere un análisis de cómo ha impactado ese primer encuentro sexual en su vida. Por último, sobre la relación de las variables de emociones y edad del debut sexual, la varianza compartida se encuentra alrededor del $30 \%$, es un porcentaje bajo por lo cual se require tomar los resultados con reservas; surge la pregunta si no habrá alguna variable mediadora o moderadora que permita tener indicadores diferentes que permitan explicar estas relaciones, por ejemplo, el concenso de la relación sexual.

Algunas de las limitaciones de la presente investigación tiene que ver con la edad de los participantes, si bien el reporte que hacen de su prime encuentro sexual es cuando ellos tenían 17 años; la muestra evaluada tiene entre de los 21 y 25 años, de tal forma que pudiese haber problemas de memoria, así los problemas propios que tiene las medidas de autoreporte (Dang King y Inzlicht, 2020); con base en ello de acuerdo con Sprecher, O'Sullivan, Drouin y Verette-Lindenbaum (2019), si bien la investigación centrada en el debut sexual suelen utilizarse diseños transversales y retrospectivos; es necesario, que se comience a utilizar diseños longitudinales para tener una mejor comprensión de cómo la primera relación sexual influye en sus próximos encuentros sexuales, de tal forma que se sugiere para futuras investigaciones, evaluar en diferentes muestras de edad, por un lado, para conocer si existen diferencias en la percepción que tienen de ese primer encuentro sexual; y por otro lado, hacer investigación en donde se dé seguimiento a las mismas personas para analizar las posibles diferencias o similitudes que pudiesen tener y cómo influye en su conducta sexual.

Otro posible limitación tiene que ver con la percepción que se tiene de las emociones, después de ocurrido el evento, así como después del tiempo transcurrido de dicho evento; si bien como menciona Levenson (1994) respecto a que una emoción si bien es una reacción psocofisiológica, en sí misma representa modos de adaptación a ciertos estímulos del individuo cuando percibe un objeto, persona, lugar, suceso o recuerdo importante; es decir, en el caso de las emociones experimentadas en la primera relación sexual, dichas emociones pueden experimentarse y mantenerse en sus futuras relaciones sexuales. Sin embargo, surge la pregunta, qué tanto cambian o se mantienen estas emociones, motivo por el cual se sugiere, en futuras investigaciones, evaluar a hombres y mujeres que hayan tenido poco tiempo de haber tenido su primer encuentro sexual, así como personas que tengan más años de haber tenido su primera relación sexual, utlizando diseños longitudinales o de cohortes de edad. 


\section{CONCLUSIONES}

Los resultados de la presente investigación, presentan las experiencias de hombres gay y mujeres lesbianas en un contexto en particular; por lo que es necesario evaluar en muestras similares, así como en personas que hayan tenido recientemente su primer encuentro sexual, para analizar si estos resultados son similares o distan entre sí, de tal forma que permita identificar cómo es que las emociones influyen en la primera relación sexual; de ahí la importancia de haber presentado los resultados del tamaño del efecto. De igual forma es necesario evaluar en población heterosexual para identificar si hay diferencias en la respuesta emocional que experimentan. También es pertinente analizar, si estas emociones tienen alguna relación con su patrón de comportamiento sexual y con su relación con conductas preventivas y de riesgo.

Los hallazgos de la presente investigación presentan análisis preeliminares de cómo las emociones pudieran tener un papel importante en el inicio de la vida sexual de hombres y mujeres de la comunidad LGBT, si bien ha transcurrido tiempo desde ese primer encuentro sexual, es necesario diseñar estrategias de intervención que permita resignificar ese primer encuentro sexual y que busquen recursos para promover conductas sexuales más seguras.

\section{Agradecimientos / Acknowledgments:}

Los autores agradecen el apoyo brindado al editor general del portal Web \#SoyHomosensual, quien autorizó la publicación del instrumento en su portal.

\section{Fuentes de financiamiento / Funding:}

Investigación realizada gracias al Programa UNAM-PAPIIT IN309719

\section{Rol de los autores / Authors Roles:}

RSM: realizó las gestiones para realizar la investigación, el diseño experimental, redacción, interpretación de resultados

SXBG: realizó los proceso los datos, búsqueda de información; interpretación de resultados y corrección del artículo

DJEN: realizó los proceso los datos; interpretación y análisis de resultados de resultados y corrección del artículo

SIMM: interpretación y análisis de resultados y corrección del artículo

\section{Aspectos éticos / legales; Ethics / legals:}

Se ha respetado lo establecido por las normativas éticas que regulan el ejercicio profesional (Código de Ética del Consejo Mexicano de Psicología). Se procuró la 
protección de la confidencialidad de la información personal, asegurando el anonimato de las personas involucradas en la muestra.

\section{Conflicto de intereses / Competing interests:}

Los autores manifestamos no haber tenido ningún conflicto de intereses al realizar esta investigación y artículo.

\section{REFERENCIAS}

Amoateng, A. Y. (2017). Does religion affect sexual behaviour? Focus on first sexual intercourse among youth in North West University South Africa. Gender \& Behaviour, 15(2), 8879-8896.

Antona, A., Madrid, J. y Aláez, M. (2003). Adolescencia y salud. Papeles del Psicólogo, 23(84), 45-53.

Arreola, S., Neilands, T., Pollack, L., Paul, J., y Catania, J. (2008). Childhood sexual experiences and adulth health sequelae among gay and bisexual men: defining childhood sexual abuse. Journal of Sex Research, 45(3), 246-252.

Bárcena, G. S., Rendón, R. G. y Robles, M. S. (2011). Factores asociados a la conducta sexual protegida: una revisión. En R. Díaz-Loving y S. Robles Montijo (Eds.), Salud y Sexualidad (pp. 5-47). México D.F.: UNAM, FES Iztacala, Asociación Mexicana de Psicología Social, Consejo Nacional de Ciencia y Tecnología.

Brown, M. J., Masho, S. W., Perera, R. A., Mezuk, B. y Cohen, S. A. (2015). Sex and sexual orientation disparities in adverse childhood experiences and early age at sexual debut in the United States: Results from a nationally representative sample. Child Abuse \& Neglect, 46, 89-102. doi:10.1016/j.chiabu.2015.02.019

Caballero, A., Carrera, P., Sánchez, F., Muñoz, D. y Blanco, A. (2003) La experiencia emocional como predictor de los comportamientos de riesgo. Psicothema. 15 (5), $427-432$.

Cárdenas, M. \& Arancibia, H. (2014). Potencia estadística y cálculo del tamaño del efecto en $\mathrm{G} *$ Power: Complementos a las pruebas de significación estadística y su aplicación en psicología. Salud \& Sociedad, 5(2), 210-224.

Carpenter, D., Janssen, E., Graham, C., Vorst, H., y Wicherts, J. (2008). Women's scores on the Sexual Inhibition=Sexual Excitation Scales (SIS=SES): Gender similarities and differences. Journal of Sex Research, 45, 36-48.

Carrier, J. (1976). Cultural factors affecting urban mexican male homosexual behavior. Archives of Sexual Behavior, 5(2), 103-124.

CENSIDA. (2017). Vigilancia Epidemiológica de casos de VIH/SIDA en México Registro Nacional de Casos de SIDA. Actualización al 1er. trimestre del 2017. Recuperado de http://www.gob.mx/cms/uploads/attachment/file/220364/RN_1er_trim_2017.pdf

Chenchang, X., Wei, W., Yue, C., Hong, Y., Shiyue, L., Jingjing, L., et al. (2017). The influence of condom use during the first-time anal intercourse on the subsequent 
sexual behaviors among young men who have sex with men in China. Biomedical Research (0970-938X), 28(19), 8537-8541.

Chiao, C. y Ksobiech, K. (2015). The influence of early sexual debut and pubertal timing on psychological distress among Taiwanese adolescents. Psychology, Health \& Medicine, 20(8), 972-978. doi:10.1080/13548506.2014.987147

Climent, G. (2009). Voces, silencios y gritos: Los significados del embarazo en la adolescencia y los estilos parentales educativos. Revista Argentina de Sociología, 7(13), 186-213.

Cummings, T., Auerswald, C. L. y Ott, M. A. (2014). Factors infl uencing abstinence, anticipation, and delay of sex among adolescent boys in High-Sexually Transmitted Infection prevalence communities. Journal of Adolescent Health, 54, 593-598.

Dang, J., King, K. \& Inzlicht, M. (2020). Why Are Self-Report and Behavioral Measures Weakly Correlated? 24(4), 267-269. https://doi.org/10.1016/j.tics.2020.01.007

Day, R. (1992). The transition to first intercourse among racially and culturally diverse youth. Journal Marriage Family, 54(4), 749-762.

Delgado, A. J., Gordon, D. y Schnarrs, P. (2016). The effect of discrimination and stress on sexual and behavioral health among sexual minority servicemen. Journal of Gay \& Lesbian Mental Health, 20(3), 258-279. doi:10.1080/19359705.2016.114344

Drury, K. M. y Bukowski, W. M. (2013). Sexual Development. En D. S. Bromberg \& W. T. O’Donohue (Eds.) Handbook of Child and Adolescent Sexuality (pp.115-144). doi:10.1016/B978-0-12-387759-8.00005-2

Fontenberry, J. D. (2013). Sexual Development in Adolescents. En D. S. Bromberg y W. T. O'Donohue (Eds.), Handbook of Child and Adolescent Sexuality (pp. 171-192). San Diego, CA, US: Elsevier Academic Press. doi:10.1016/B978-0-12-387759-8.00007-6

Gilliam, M. L., Berlin A., Kozloski M., Hernandez M. y Grundy, M. (2007). Interpersonal and personal factors influencing sexual debut among Mexican-American young women in the United States. Journal of Adolescent Health, 41, 492-503.

Goldmeir, D., y Richardson, D. (2005). Romantic love and sexually transmitted infection acquisition: Hypothesis and review. International Journal of STD \& AIDS, 16(9), 585-587.

Gómez, F. y Barrientos, J.E. (2012). Efectos del prejuicio sexual en la salud mental de gays y lesbianas, en la ciudad de Antofagasta, Chile. Sexualidad, Salud y SociedadRevista Latinoamericana, 100-123.

Gravel, E., Young, M., Darzi, C., Olavarria-Turner, M. y Ming-Lee, A. (2016). Premarital Sexual Debut in Emerging Adults of South Asian Descent: The Role of Parental Sexual Socialization and Sexual Attitudes. Sexuality \& Culture, 20(4), 862-878. doi:10.1007/s12119-016-9362-1

Herek, G. (2007). Sexual orientation and mental health. The annual Review of Clinical Psychology, 3, 353-375. 
INEGI. (2010). Encuesta Nacional de la Dinámica Demográfica 2009. Recuperado de http://internet.contenidos.inegi.org.mx/contenidos/Productos/prod_serv/contenidos/ espanol/bvinegi/productos/metodologias/est/informe_op_enadid09.pdf

Iniewicz, G., Sałapa, K., Wrona, M. y Marek, N. (2017). Minority stress among homosexual and bisexual individuals - from theoretical concepts to research tools: The Sexual Minority Stress Scale. Archives of Psychiatry \& Psychotherapy, 19(3), 69-80. doi:10.12740/APP/75483

Juárez, F. y Gayet, C. (2005). Salud sexual y reproductiva de los adolescentes en México: Un nuevo marco de análisis para la evaluación y diseño de políticas. Papeles de la población, 45, 177-219.

Kar, S. K., Choudhury, A. \& Singh, A. P. (2015). Understanding normal development of adolescent sexuality: A bumpy ride. Journal of Human Reproductive Sciences, 8(2), 70-4. doi: 10.4103/0974-1208.158594

Kastbom, Å. A., Sydsjö, G., Bladh, M., Priebe, G. y Svedin, C. G. (2015). Sexual debut before the age of 14 leads to poorer psychosocial health and risky behaviour in later life. Acta Paediatrica, 104(1), 91-100. doi:10.1111/apa.12803

Kerlinger, F. y Lee, H. (2002). Investigación del comportamiento. Métodos de investigación en ciencias sociales (4a. ed.). México: McGraw Hill.

Lagunes, R. (2017). Recomendaciones sobre los procedimientos de construcción y validación de instrumentos y escalas de medición en la psicología de la salud. Psicología y Salud, 27(1), 5-18.

Lai Tong Lee, R., Loke, A. Y., Tsz Man Hung, T. y Sobel, H. (2018). A systematic review on identifying risk factors associated with early sexual debut and coerced sex among adolescents and young people in communities. Journal of Clinical Nursing, 27(3/4), 478-501. doi:10.1111/jocn.13933

Lazarus, R. (1998). Fifty Years of the Research and theory of R.s. Lazarus: An Analysis of Historical and Perennial Issues. New Yersey: Psychology Press.

Levenson, R.W. (1994). Human emotion. A funtional view. En: P. Ekman \& R.J. Davidson (Eds). The nature of Emotions: Fundamental Questions, (pp.123-126). New York: Oxford University Press.

Lieberman, L. D., Goldfarb, E. S., Kwiatkowski, S. y Santos, P. (2017). Does First Sex Really "Just Happen?” A Retrospective Exploratory Study of Sexual Debut Among American Adolescents. American Journal of Sexuality Education, 12(3), 237-256. do i:10.1080/15546128.2017.1359804

Longmore, M. A., Manning, W. D. y Giordano, P. C. (2001). Preadolescent parenting strategies and teens'dating and sexual initiation: A longitudinal analysis. Journal of Marriage and Family, 63(2), 322-335.

Lowry, R., Dunville, R., Robin, L. y Kann, L. (2017). Early Sexual Debut and Associated Risk Behaviors Among Sexual Minority Youth. American Journal of Preventive Medicine, 52(3), 379-384. doi:10.1016/j.amepre.2016.10.008 
Lowry, R., Johns, M. M., Robin, L. E. y Kann, L. K. (2017). Social Stress and Substance Use Disparities by Sexual Orientation Among High School Students. American Journal of Preventive Medicine, 53(4), 547-558. doi:10.1016/j.amepre.2017.06.011

Magnusson, B. M., Nield, J. A. y Lapane, K. L. (2015). Age at first intercourse and subsequent sexual partnering among adult women in the United States, a cross-sectional study. BMC Public Health, 15(1), 1-9. doi:10.1186/s12889-015-1458-2

Malhotra, S. (2008). Impact of the sexual revolution: Consequences of risky sexual behaviors. Journal of American Physicians and Surgeons, 13(3), 88-90.

Miner, M. H., Dickenson, J., \& Coleman, E. (2019). Effects of Emotions on Sexual Behavior in Men with and without Hypersexuality. Sexual addiction \& compulsivity, 26(1-2),24-41. https://doi.org/10.1080/10720162.2018.1564408

Miño-Worobiej, A. (2008) Imágenes de género y conductas sexual y reproductiva. Salud Pública de México, 50(1), 17-31.

Nigatu, D. T., Seme, A., Fituma, S. y Segni, M. T. (2018). Sexual initiation and associated factors among young women in West Shoa, Ambo Town, Ethiopia: a community-based cross-sectional study. BMC Women's Health, 18(1), N.PAG-N.PAG. doi:10.1186/ s12905-018-0563-7

ONUSIDA (2016). Hoja informativa - últimas estadísticas sobre el estado de la epidemia de sida. Recuperado de http://www.unaids.org/es/resources/fact-sheet

Organización Panamericana de la Salud / Organización Mundial de la Salud (2000). Promoción de la salud sexual. Recuperado de: http://www.e-sanitas.edu.co/Diplomados/ VIH/Modulo\%201/lecturas/Promocion_de_Salud_Sexual\%20OMS\%202000.pdf

Piña, J. A., González, D., Molina, C. y Cota, M. (2003). Variables psicológicas y VIH/ SIDA en estudiantes de educación superior: estudio comparativo en función del sexo y de la edad. Revista Colombiana de Psicología, 12, 19-27.

Pollard, A., Nadarzynski, T. y Llewellyn, C. (2018). Syndemics of stigma, minority-stress, maladaptive coping, risk environments and littoral spaces among men who have sex with men using chemsex. Culture, Health \& Sexuality, 20(4), 411-427. doi:10.1080/1 3691058.2017.1350751

Reissing, E.D., Andruff, H. L. y Wentland, J. J. (2012). Looking Back: The Experience of First Sexual Intercourse and Current Sexual Adjustment in Young Heterosexual Adults. Journal of Sex Research, 49(1), 27.35. doi: 10.1080/00224499.2010.538951

Riggs, D. W. y Treharne, G. J. (2017). Decompensation: A Novel Approach to Accounting for Stress Arising From the Effects of Ideology and Social Norms. Journal of Homosexuality, 64(5), 592-605. doi:10.1080/00918369.2016.1194116

Sánchez, R., Enríquez, D. \& Rosales, R. (2019). Información, motivación y habilidades conductuales asociadas al uso del condón en la última relación sexual en hombres privados de la libertad. Revista Persona, 22(1), 37-52. doi: 10.26439/persona2019. n022(1).4082 
Sattler, F. A., Franke, G. H. y Christiansen, H. (2017). Mental health differences between German gay and bisexual men and population-based controls. BMC Psychiatry, 17, 1-7. doi:10.1186/s12888-017-1435-7

Shrestha, R., Karki, P. y Copenhaver, M. (2016). Early Sexual Debut: A Risk Factor for STIs/HIV Acquisition Among a Nationally Representative Sample of Adults in Nepal. Journal of Community Health, 41(1), 70-77. doi:10.1007/s10900-015-0065-6

Sprecher, S., O’Sullivan, L.F., Drouin, M. y Verette-Lindenbaum, J. (2019). The Significance of Sexual Debut in Women's Lives. Current Sexual Health Reports, 11, 265273. doi: https://doi.org/10.1007/s11930-019-00228-5

Tenkorang, E. Y. y Maticka-Tyndale, E. (2014). Individual- and Community-Level Influences on the Timing of Sexual Debut Among Youth in Nyanza, Kenya. International Perspectives on Sexual \& Reproductive Health, 40(2), 68-78. doi:10.1363/4006814

Trenchard, L. y Warren, H. (1984). Something to tell you. London: London Gay teenage Group.

Upchurch, D. M., Mason W. M., Kusunoki Y. y Kriechbaum, J. (2004). Social and behavioral determinants of self-reported std among adolescent. Perspectives on Sexual and Reproductive Health, 36(6), 276-8.

Vall-Mayans et al. (2006). Reemergencia de la sifilis infecciosa en varones homosexuales y coinfección por el virus de inmunodeficiencia humana en Barcelona, 2002-2003. Medicina Clínica, 126(23), 94-96.

Vargas, E. y Barrera, F. (2002). Adolescencia, relaciones románticas y actividad sexual: una revisión. Revista Colombiana de Psicología, (11), 115-134.

Vargas, E., Henao, J. y González, C. (2007). Toma de decisiones sexuales y reproductivas en la adoelscencia. Acta Cololombiana de Psicología, 10(1), 49-63.

Vega, Z., Muñoz. S., Berra, E., Nava. C. y Escobar, G. (2012). Identificación de emociones desde el modelo de Lazarus y Folkman: propuesta del cuestionario de estrés, emociones y afrontamiento (CEEA). Revista Electrónica de Psicología Iztacala, 15(3), 1051-1073.

Vicuña, J. (2017). Prevalencia de ansiedad y depresión en la población no heterosexual del cantón Quito 2016. (Especialidad en Psiquiatría), Universidad Central del Ecuador.

Whitley, B. (2001). Gender-role variables and attitudes toward homosexuality. Sex roles, 45(11), 671-719. 